The way in which this may be accomplished will be best shown by detailing a case and its treatment:-

A lady in Northamptonshire had long suffered from anasarca, the result of heart-disease. From the chest downwards the body was so much distended with fluid that for many montha she had been unable to lie down, but was obliged to sit day and night on a chair. On account of her size and weight, every requisite movement was effected with difficulty; her breathing was short and laboured: to be brief, her death was almost from hour to hour expected. At a consultation on the case, Dr. Robertson (consulting physician to the Northampton Infirmary), Mr. Dix (of Long Buckby), and myself being present, the following mode of relief was adopted in the utter hopelessness of any other:-Each leg was placed in a separate foot.bath, when a lancet was deeply inserted into the mass of cellular tissue, which projected most on the outer side of each ankle. A stream of water instantly began to flow from both apertures, and continued to do so with such volume and rapidity that in less than twelve hours the whole of it was removed, and the body and legs had assumed their natural size and form. Bandages of flannel were repeatedly applied as the diminishing size required, and were kept tight about the kody as a precaution against faintness. The relief to the patient was immediate and gratifying, and for the first time for several months she lay down on her bed and slept comfortably. There was, of course, a slight oozing from the apertures; but these, being kept closed by adhesive plaster and bandages, readily healed-almost as soon, perhaps, as they would have done in a patient otherwise healthy. The operation was subsequently performed by $\mathrm{Mr}$. Dix six or seven times during the remainder of the lifetime of this patient, which lasted about five months after the first ad. ministering of relief, and the water was never again allowed to accumulate so as to cause inconvenience.

I trust that a satisfactory proof is here given of what may be safely done, even in the advanced stage of anasarea; and I submit that the same treatment will prove efficacious in its earlier stages by the removal of smaller accumulations of water, while the operation may be repeated as often as is required. Some faintness occurred after the first operation in the above ease; indeed, the loss of a large quantity of fluid which has so long pressed on the bloodvessels must inevitably cause this tendency. Hence arises the necessity of keeping a bandage round the body; and it is necessary also that the patient should keep strictly quiet for some time. As to erysipelas following this operation (the probability of which has been suggested by one or two of my medical friends), I am not of opinion that this is much to be feared. Let me add, that if patients afflicted with anasarca are to be left without artificial aid in the dis. charge of the serum collected, - what follows? Nature attempts it, in a somewhat similar, but ineffectual, manner; the distension goes on increasing, till at last the legs burst open by ulceration, and then only a partial escape of the fluid takes place; no real relief ensues, not to speak of the pain of so tedious a process. In the majority of cases, I believe that eertain relief may be given, as well as life prolonged.

Spring gardens, Oet. 1861.

\section{SUCCESSFUL OPERATION}

$$
\text { FOR }
$$

\section{THE CURE OF VESICO-VAGINAL FISTULA WITH IRON-WIRE SUTURES.}

By JOHN JAMES, I.R.C.S.I.,

OEDICAI OFFICER TO KILEENNY UNION INFTRMTART AYD FETER HOSPITAL, SUGgEON TO THE COUNTY GAOL, ETC.

C. B-_, aged twenty-six years, admitted into Kilkenny Union Infirmary May 27th, 1861. Was confined of her first child on the 24th of April last, after prolonged and tedious labour, but without instrumental aid. Sloughing of the bladder followed, and complete incontinence of urine was the result. At the date of her admission every drop of urine escaped in voluntarily, whatever position she might be in.

Upon examination, an opening was found in the fundus of the bladder, which would admit the largest-sized catheter with ease. The margins were soft and unindurated. Externally,

he parts were excoriated by the constant dribbling away of he urine.

determined to attempt to relieve her from the distressing condition she was in by an operation with iron-wire sutures, as practised by Dr. Simpson; and on the 27 th of June (her bowels having been previously acted on by an enema) I proceeded to operate, assisted by my colleague, Dr. Potter, and Drs. Kinchella and Fitzsimons, who kindly co-operated on the occasion.

The patient was placed on her knees and face on the operating table; consequently no chloroform was used. The vagina being then dilated with the duck-bill speculum, held by an assistant, I took hold of the lower edge of the fistula with a tenaculum, and pared off about $t$ wo lines in breadth of mucous membrane round the opening with a straight spear shaped knife; and having carefully examined the cut surfaces with the help of a convex lens about four inches in diameter, which I found very useful for the purpose, removed every portion of mucons membrane likely to interfere with the success of the operation.

After a delay of a few minutes to allow the bleeding to cease, I inserted three iron-wire sutures with the tubular needle, assisted by the blunt hook, supporting them while being drawn out on the fork invented for the purpose by Bozeman; and having pressed together the raw surfaces with the finger of the left hand, while the wires were held tightly in the right, I passed along the sutures the twisted-wire splint, prepared as recommended by Dr. Simpson, and made it firm against the wall of the vagina by twisting each pair of wires over the lower bar. The cut edges were found by this means to be kept immovably fixed, and in close apposition. The patient was now removed to bed, and a metallic catheter, of the usual sigmoid shape, placed in the bladder. Some irritability of stomach and vomiting ensued. She was directed to take one grain of opium every third hour, and to have a milk diet.

(I may here mention, that the bed used in this case was con. structed on the plan contrived by Dr. Corrigan, the web bottom being covered with folded blankets, so arranged that an opening was left in the centre between two of the bands, protected from wet by gutta-percha tissue, through which the urine drained away to a vessel placed underneath, without any in. convenience to the patient. )

June 28th. - She passed a good night; no urine escaped through the fistula; the catheter was removed night and morning, and the vagina syringed with tepid water.

$30 \mathrm{th}$. - Going on well; no escape of urine.

July lst.-Her bowels were freely moved three times to-day, and in consequence the catheter came away; but no urine escaped until it was replaced. She continued to progress favourably until the $5 t h$, when the catheter, becoming irksome from its weight, was replaced by a gum elastic one of the same form, kept in shape by a slender wire stilette, and she expressed herself much relieved by the change.

6th. - This being the ninth day, the splint was removed by dividing the sutures with scissors under the lower bar and raising it up, when the edges of the opening were found to be perfestly united. The catheter was kept in for two days longer; after which she was directed to pass urine at short intervals at first, which she did without pain or inconvenience.

20 th. - No escape of urine through the fistula since the operation.

The patient remained in hospital until July 31st, when she was discharged, being perfectly free from all trase of her former complaint.

Kilkenny, 1861.

Vaccination through Cow's Milk.-M. Soubie, of Libourne (France), states, in the Gasette des Hôpitaux of the 10th instant, that he vaccinated the teats of a cow, and obtained two fine vesicles. The milk of this cow was given to two children, one six months old and being brought ap by hand, the other fourteen months and weaned. The first took this milk for two days, on the fifth and sixth day of the vaccination of the cow; the second drank it only one day, on the eighth day of the vaceination. This latter child consumed about ten ounces of the milk; the first, nearly double that quantity. One month after this experiment the two children were vaceinated in the usual manner, but with a negative result; whilst the same lymph used with them acted very fully upon another child vaccinated at the same time. M. Soubie was induced to try this indirect mode of vaccination by a case in which a mother who was suckling an infant, and who was attacked with small-pox, continued to nurse her child, the latter becoming affected with slight fever without eruption. At two and fivelyears old this child was attempted to be vaceinated, without result; and even at sixteen, vaccination proved of no effect. So M. Soubie inferred that this child had been protected by the milk of the mother. 